\title{
Compact Ultra-Wideband Double-Ridged Horn Antennas for Medical Imaging
}

\author{
Siamak Sarjoghian, Yasir Alfadhl, and Xiaodong Chen \\ School of Electronic Engineering and Computer Science \\ Queen Mary University of London \\ London E1 4NS, UK \\ s.sarjoghian@qmul.ac.uk
}

\begin{abstract}
This paper presents novel designs, analyses, and simulations of two miniaturised pyramidal double-ridged horn (PDRH) microwave antennas for medical imaging systems. The PDRH antennas have been optimised to operate within a ceramic material filling with permittivity of 41 (which approximately is the permittivity of the human skin in the intended frequency range), in order to make the compact antennas, and also to minimise the first bulky reflection due to the large contrast of the pulse from the free-space to the human skin surface, which cause the difficulty to process the overall reflected pulse. The proposed antennas can operate in conjunction with each other to cover the whole radio frequency (RF) range of 1.25 to $2.47 \mathrm{GHz}$, where the high-gain and directivity for medical imaging systems are the main factors. Both RF antennas have the bandwidth larger than $500-\mathrm{MHz}$ that would qualify them as ultra-wideband (UWB) antennas. The optimised designed microwave antennas are numerically verified based on the electromagnetic (EM) method of finite integration technique (FIT), using the CST Microwave Studio (CST-MWS) software.
\end{abstract}

Keywords-Ceramic; medical imaging; PDRH antenna; UWB.

\section{INTRODUCTION}

Ultra-wideband (UWB) signals and systems can provide a number of promising advantages in employing radiowaves for transferring the information over bandwidths of larger than 500$\mathrm{MHz}$. This technology is originally aimed at the short-range and indoor wireless applications, with the low-power consumption. It utilises a large portion of the EM spectrum in order to improve the speed, and to reduce the data transfer interference, compared to other available technologies. The independent governmental regulator and competition authority for the UK communications industries (Ofcom), and also the US Federal Communications Commission (FCC) regulation for UWB allocates the regulation for the technology that allows for the unlicensed RF operation within the range of $3.1-10.6 \mathrm{GHz}$, and the transmit power of 41.3-dBm/MHz [1].

In this technique, the signals diffuse across a wide range of frequencies in order to detect a subject accurately. It has high data rate in comparison with the other wireless technologies that enable for this method to send trains of signal pulses containing hundreds of millions of pulses per second. As it is small and very low-power, it fits the requirement to be employed as the indoor applications. In the last few years, the UWB technology is found to be a potential candidate for the medical imaging purposes, compared to the other scanning technologies, due to the unique properties that this technology offers, including:
High data rates: The large bandwidth occupied by the UWB $(<500 \mathrm{MHz})$ gives potential of speeds over $500 \mathrm{Mb} / \mathrm{s}$ and higher speed over shorter distances.

Low-cost: UWB is a low-power technology, therefore low-cost technique where the capability to adjust a pulse onto an antenna is possibly the same as a simple transmitter that consequently makes the transceiver enormously cheaper to manufacture.

Multipath immunity: UWB pulses can supply high-resolutions of reflected pulses at the receiver due to the narrow-shaped RF pulses, as a result of its capability of pulse separation. This is essential in the wireless channels due to pulses interfering each other, as the major complication to error-free systems.

Ranging and communication at the same time: Using UWB technique provides user both precise ranging (object location) and high speed data communication in the same device presents intriguing possibilities for new applications.

Replacing the ionising methods: Ionising radiation is the main concern of the scanning technologies, the UWB is identify as a good candidate for scanning as it can eliminate ionising effect compared with other RF techniques currently being used due to its short pulses used by this technology [2].

More research and activities on breast, skin, blood cancer, and head stroke detection in recent years have proved that the microwave imaging systems and techniques are used as the safe methods, but require a considerable attention. The method of using UWB techniques for detecting cancer cells suggests this technique can also be employed in order to detect the fat thickness in all the body organs such as the abdomen [3-6].

To be able to transmit an UWB pulse to the mediums and receiving the reflected pulse, there is a necessity of transmission lines for the pulse to be able to propagate. Although an UWB antenna is found as a good candidate for a human body based on its rewards, such as wide bandwidth, but yet the directivity, size reduction, and coupling effect of a human body to the antenna characteristics, are still of the primary research concerns.

In the case of using an UWB antenna as a transceiver, the chosen antenna has to satisfy the system requirements, including the high directivity and gain, wide bandwidth, and low centre frequency. There are a number of important factors that are taken into account in order to achieve the operational antennas for the intended objectives. 
Bandwidth: The first important factor in the antenna design is the bandwidth for a medical imaging system, which plays more important rule, as it can increase the image resolution.

Centre frequency: The second main factor of the design is the centre frequency of the antenna, which relates to the penetration depth capability of the pulse transmitted by into a human body.

Directivity and Gain: The most important factor in any antenna design is the directivity and gain, that in the case of the human body being vital, with respect to the propagation direction, along with the antenna gain.

Several different types of the UWB antennas are currently available in literature for human tissues scanning, some of these designs were studied because they had the elements that could satisfy the mentioned antenna requirements for the development of the medical imaging systems, such as the horn, wide-slot, Vivaldi, and planar structures [7-9].

In this paper, UWB horn antennas have been selected based on its innovative parameters such as good directivity, gain, wide bandwidth, and ease of construction. The UWB horn antenna has different types, such as conical horn, pyramid horn, doubleridged horn, and double-ridged guided horn.

The PDRH antenna has been selected and designed for two different frequencies in a material with a high permittivity of 41 that is matched with the skin permittivity, using the CST-MWS software [10].

\section{Antenna Design AND Modelling}

This section thoroughly discusses the antenna designs:

\section{A. Design Parameters}

The designs for both of the proposed antennas have been finalised within the medium with the permittivity of 41 , with respect to the permittivity of the human skin, which is found to be approximately 39.5 in the dry condition, and 43 in the wet condition within the frequency of interest (1.2 to $2.4 \mathrm{GHz})$ [11]. There are advantages that makes this design novel, as follows:

Firstly, the employed design technique (i.e., designing the antenna within the high permittivity medium), results in the compact antenna development, based on the concept that a wavelength will be reduced with respect to the reduction of a speed of a pulse in a medium with increasing the permittivity.

Secondly, any human body coupling effect to the antenna characteristics will be reduced to a minimum, with respect to the ceramic permittivity that has been chosen to be used that is close to the human skin permittivity in the specified frequency range.

Finally, using such a technique, results in the dissolve of the first large reflection of the skin, concerning the contraction of the free-space to the skin medium with the high permittivity of 39.5-43. Also, it reduces the operating frequency of the antennas which further improves the capability of the pulse to penetrate more inside the human body [12].

Table 1, shows the dimensions of both the designs that have been calculated and finalised in the progress, using the general horn equations to design the pyramidal horn antennas [13].
Two microwave antenna designs shown in Figs. 1 (a) and (b) have been designed by the aluminium and the given dimensions. The first design has k-type SMA connector that has air as the dielectric with a copper centre core that has a diameter size of $0.5 \mathrm{~mm}$, and dielectric diameter size of $1.15 \mathrm{~mm}$, and the second design has SMA connector that has Teflon dielectric with core diameter size of $0.6 \mathrm{~mm}$ and dielectric diameter size of $2 \mathrm{~mm}$.

\section{B. Far-Field Region}

Based on the given dimensions illustrated in Table 1, both designs have compact size and biggest dimension as $35 \mathrm{~mm}$ which are the long length of the antennas. With regards to the far-field and near-field regions of the microwave antennas that called Fraunhofer distance, the following equation (1) has been employed in order to calculate the distance where the antennas have to get placed with respect to the human body.

$$
d_{f}=\frac{2 D^{2}}{\lambda}
$$

where $d_{f}$ defines the far-field distance that has been calculated for the first design to be higher than $70 \mathrm{~mm}$, and for the second design higher than $100 \mathrm{~mm}$, based on the equation (1). As the radiation point is the connector core, this can be implemented by placing a $35 \mathrm{~mm}$ and $50 \mathrm{~mm}$ ceramic distances for the first and second antenna designs between the antennas and human body skin, respectively. In this case as some of the energy will be lost within the ceramic, but still there is good energy left over for the penetration through human body as the frequency region is quite low, and the selected ceramic has low-conductivity which makes the design special for the medical imaging systems.

Table1. Design dimensions of the proposed PDRH microwave antennas.

\begin{tabular}{|c|c|c|}
\hline Antenna Dimensions & First Design & Second Design \\
\hline Aperture $X$ - and $Y$-axis (mm) & $32 \times 26$ & $30 \times 30$ \\
\hline Aperture length $(\mathrm{mm})$ & 33.24 & 28.74 \\
\hline Waveguide $X$ - and Y-axis (mm) & $11.7 \times 5.85$ & $9.79 \times 5.29$ \\
\hline Waveguide length ( $\mathrm{mm})$ & 8 & 6.5 \\
\hline Angle of waveguide with aperture & $108.08^{\circ}$ & $115.23^{\circ}$ \\
\hline Height and thickness of ridges $(\mathrm{mm})$ & $1.92 \times 3$ & $1.5 \times 3$ \\
\hline Antenna thickness (mm) & 1 & 0.105 \\
\hline 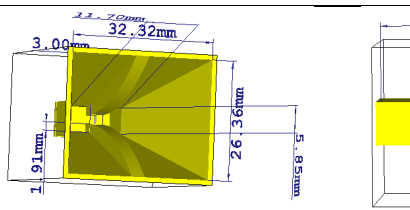 & & \\
\hline
\end{tabular}

Fig. 1 (a). Simulated Design of the $1^{\text {st }}$ PDRH microwave antenna.
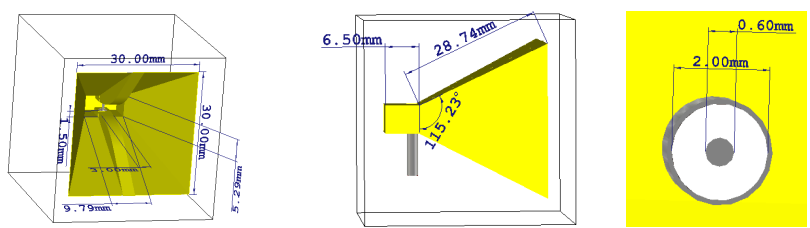

Fig. 1 (b). Simulated design of the $2^{\text {nd }}$ PDRH microwave antenna. 


\section{MicrowaVe ANTENNAS Simulations}

In this section, the pair of PDRH microwave antennas have been accurately designed and simulated in the CST-MWS, and simulation results have been discussed in detail.

\section{A. Simulation and Analysis of the First PDRH Antenna}

The first PDRH antenna design, which the dimensions are given in Table 1, has the bandwidth of approximately $550-\mathrm{MHz}$ in $-10 \mathrm{~dB}$ point. This has been illustrated in the $S_{11}$ graph shown in Fig. 2 (a), that starts from $1.25-\mathrm{GHz}$ and ends at $1.8-\mathrm{GHz}$, the highest deep pick point in the $S_{11}$ graph is approximately at 1.4$\mathrm{GHz}$ which goes down to $-38.8 \mathrm{~dB}$. With respect to the far-field radiation pattern that has been generated using the CST-MWS and is shown in the Fig. 3 (a), the antenna has good directivity, small back lobes, and gain of 8.72-dB, at 1.4-GHz. Fig. 3 (a) also presents that the antenna has been linearly polarised in both the $E$-field and $H$-field regions. The gain over the entire bandwidth frequencies has been generated by the CST-MWS that is shown in Fig. 4 (a), which depicts that the gain will be increased by moving to the higher frequencies. According to the far-field distance, which had been already defined to reduce the human body coupling effect to minimum, the distance calculated to be greater than $35 \mathrm{~mm}$ from the antenna aperture, the antenna can further be used for any imaging system where penetration inside the human body is of the concerns, with respect to the antenna's low centre frequency; e.g., in breast cancer and head stroke.

\section{B. Simulation and Analysis of the Second PDRH Antenna}

The second antenna design with the given dimensions as in Table 1, has bandwidth of approximately $700-\mathrm{MHz}$ in $-10 \mathrm{~dB}$ point. This has been shown in the $S_{11}$ graph, which is presented in Fig. 2 (b), the bandwidth starts from 1.76-GHz and ends at 2.47-GHz. The highest deep pick point in the $S_{11}$ graph is at 1.95 $\mathrm{GHz}$, which goes down to $-22.65 \mathrm{~dB}$ point. With regards to the far-field radiation pattern, as shown in Fig. 3 (b), the antenna has good directivity, small side, and back lobes, and high gain of 12 $\mathrm{dB}$ in the $1.975 \mathrm{GHz}$ point. Also, the Fig. 3 (b) shows that the antenna has been linearly polarised in both the $E$-field and $H$ field. The antenna gain over the entire frequencies of bandwidth has been generated by the software that is shown in Fig. 4 (b). By reviewing the graph, it is also noticeable that the gain will be increased slightly by moving towards the higher frequencies.

Concerning the far-field distance, which is set to be more than $50 \mathrm{~mm}$ from the aperture of the antenna to the skin surface, which further presents the technique has not only filled the gap between the antenna and the detecting tissue, but also, it reduces the distance between both, with respect to reducing the coupling effect which may alter the characteristics. The antenna can be employed for any imaging system where penetration inside the human body is not of the main concern with respect to the higher centre frequency of the antenna, compared to previous design. Bear in mind the bandwidth of the antenna is much greater than previous design which enables user to transmit narrower pulse, and therefore higher resolution compared to previous design and thinner layer of the human tissues is subject to the detection and analysis, such as skin cancer and abdominal fat detection [14].

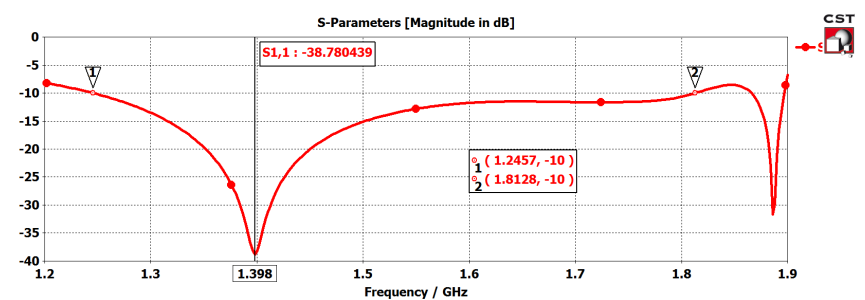

Fig. 2 (a). $S_{11}$ plot in the frequency range of $1.2-1.9 \mathrm{GHz}$, for the $1^{\text {st }}$ design.

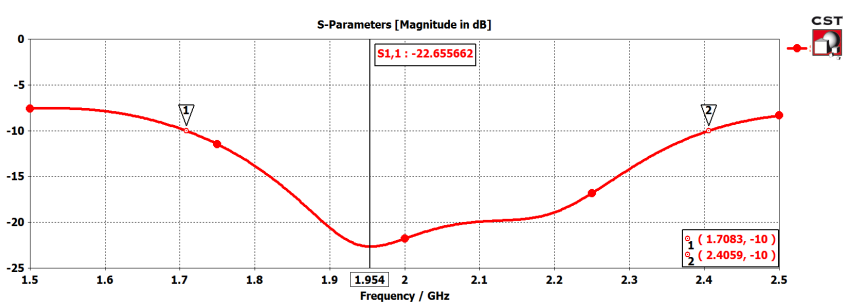

Fig. 2 (b). $S_{11}$ plot in the frequency range of $1.5-2.5 \mathrm{GHz}$, for the $2^{\text {nd }}$ design.
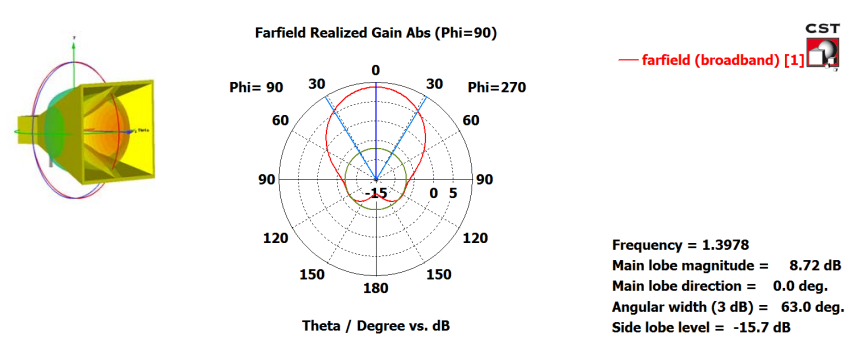

Fig. 3 (a). Simulated far-field EM radiation pattern for the $1^{\text {st }}$ design.
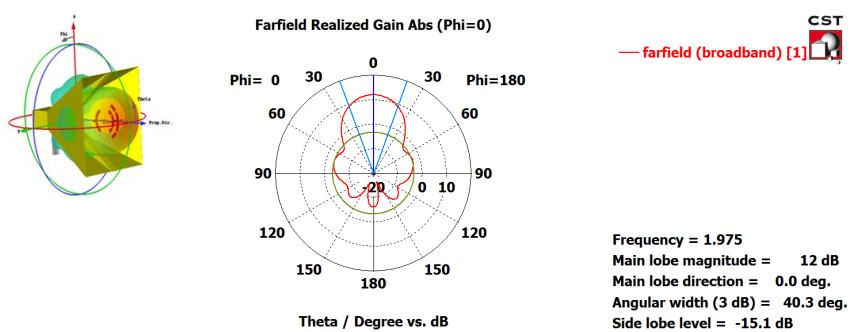

Fig. 3 (b). Simulated far-field EM radiation pattern for the $2^{\text {nd }}$ design.

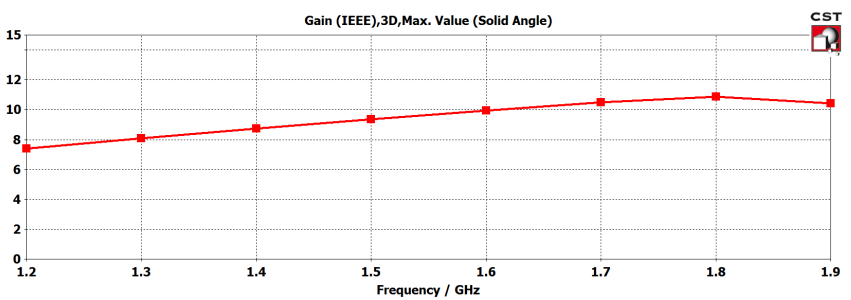

Fig. 4 (a). Antenna gain over the bandwidth frequency, for the $1^{\text {st }}$ design.

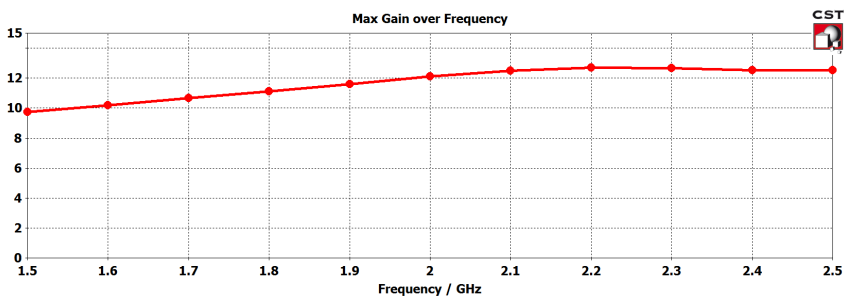

Fig. 4 (b). Antenna gain over the bandwidth frequency, for the $2^{\text {nd }}$ design. 


\section{DISCUSSION}

The antennas design and simulations are in well-agreement according to the following technical concerns:

Both antennas have bandwidth larger than 500-MHz, which is the principle for an UWB antenna category. They can cover bandwidth of $1.2-\mathrm{GHz}$ which starts from 1.25 to $2.45 \mathrm{GHz}$. However, there are needs for switches in order to change from one antenna to another in the case of using different frequencies.

The inclusion of the high-permittivity material (i.e., ceramic) within the antenna is important, not only for scaling down the antenna size, but also to minimise the huge reflection resulting from the mismatch between the free-space and the skin surface, as well as filling the gap between them. The presented technique also shortens the distance between the antenna and the human body based on minimising the coupling effect that can alter the antenna characteristics.

The proposed technique had pushed the centre frequency to the lower range; however, the bandwidth had been deduced as it was expected, but is still more than $30 \%$ of the centre frequency. The antennas inside the high-permittivity medium, have also shown the high-performance in terms of the gain and directivity.

\section{CONCLUSION}

The two miniaturised horn microwave antennas have been accurately designed, optimised, and tested for reflection-based fat-thickness detection systems. Both the horn antenna designs were embedded with the skin permittivity equivalent, ceramic material, to miniaturise the RF designs, and to shift the required centre frequency to the lower frequency value, which increases the penetration depth inside the human body. The use of highpermittivity filling has also resulted in the reduction of the pulse bandwidth. The proposed antenna designs can in fact eliminate the difficulty of having a large reflection pulse when the pulse radiated in the free-space hits the human skin surface with high- permittivity, which further results in the energy loss, as well as the difficulty to retrieve the reflected pulse back from the tissue.

\section{REFERENCES}

[1] F. C. Commission, "Revision of part 15 of the commission's rules regarding ultra-wideband transmission systems, FIRST REPORT AND ORDER," ET Docket 98-153, FCC 02-48, pp. 100-118, 2002.

[2] M. Ghavami, L. Michael, and R. Kohno, Ultra Wideband Signals and Systems in Communication Engineering, $2^{\text {nd }}$ Edition, John Wiley \& Sons, 2007.

[3] M. Klemm et al., "Experimental and clinical results of breast cancer detection using UWB microwave radar" in IEEE Antennas and Propagation Society International Symposium, Jul. 2008.

[4] M. A. A. Eldosoky, "The ability of ultra wideband signals in detection of the Skin Tumour" in URSI Assembly and Scientific Symp., 2008.

[5] M. A. A. Eldosoky and H. M. Moustafa, "Detection of the blood Leukemia by using the ultra wide band pulses" in XXXth URSI General Assembly and Scientific Symposium, Aug. 2011.

[6] A. Fhager and M. Persson, "Stroke detection and diagnosis with a microwave helmet" in $6^{\text {th }}$ European Conference on Antennas and Propagation (EuCAP), Mar. 2012, pp. 1796-1798.

[7] S. A. Ruzdiana, An UWB Antenna Array for Breast Cancer Detection, Master's Thesis, University of Gavle, Sweden, Mar. 2011.

[8] H. Zhang et al., "A smart antenna array for brain cancer detection" in Loughborough Antennas and Propagation Conf. (LAPC), Nov. 2011.

[9] M. Mokhtaari and J. Bornemann, "Directional ultra-wideband antennas in planar technologies" in $38^{\text {th }}$ European Microwave Conference (EuMC), Oct. 2008, pp. 885-888.

[10] CST MICROWAVE STUDIO, EM Simulation Software 2016 [Online].

[11] IFAC-CNR. Dielectric Properties of Body Tissues. [Online] Available: http://niremf.ifac.cnr.it/tissprop/

[12] U. Schwarz et al., "Design and application of dielectrically scaled doubleridged horn antennas for biomedical UWB radar applications," in IEEE International Conf. on Ultra-Wideband (ICUWB), Sep. 2009.

[13] C. A. Balanis, Antenna Theory: Analysis and Design, $3^{\text {rd }}$ Edition, John Wiley \& Sons, Inc., May 2005.

[14] S. Sarjoghian, Y. Alfadhl, X. Chen, "A novel wide-band reflection-based system for measuring abdominal fat in humans," in URSI Commission B Int. Symp. on Electromagnetic Theory (EMTS), Aug. 2016. 